\title{
Random Matrix Models for the Hermitian Wilson-Dirac operator of QCD-like theories
}

\author{
Mario Kieburg \\ Department of Physics and Astronomy, State University of New York at Stony Brook, NY \\ 11794-3800, USA \\ E-mail: mario.kieburgestonybrook.edu
}

\section{Jacobus J. M. Verbaarschot}

Department of Physics and Astronomy, State University of New York at Stony Brook, NY 11794-3800, USA

E-mail: jvechi.physics.sunysb.edu

\section{Savvas Zafeiropoulos*}

Department of Physics and Astronomy, State University of New York at Stony Brook, NY 11794-3800, USA

E-mail: szafeiro@ic.sunysb.edu

\begin{abstract}
We introduce Random Matrix Models for the Hermitian Wilson-Dirac operator of QCD-like theories. We show that they are equivalent to the $\varepsilon$-limit of the chiral Lagrangian for Wilson chiral perturbation theory. Results are obtained for two-color QCD with quarks in the fundamental representation of the color group as well as any-color QCD with quarks in the adjoint representation. For $N_{c}=2$ we also have obtained the lattice spacing dependence of the quenched average spectral density for a fixed value of the index of the Dirac operator. Comparisons with direct numerical simulations of the random matrix ensemble are shown.
\end{abstract}

The 30th International Symposium on Lattice Field Theory

June 24-29, 2012

Cairns, Australia

${ }^{*}$ Speaker. 


\section{Introduction}

Chiral Random Matrix Theories [1,2] have been successful in describing lattice QCD Dirac spectra on the scale of the eigenvalue spacing. It was shown that they are equivalent to the $\varepsilon$-limit of QCD which is given by the $\varepsilon$-limit of chiral perturbation theory [3]. Recently, Random Matrix Theory was extended to include discretization effects of both the Wilson [4] and the staggered Dirac operator [5]. They are equivalent to the $\varepsilon$-limit of Wilson chiral perturbation theory [6, 7] and staggered chiral perturbation theory, respectively [8].

Starting from the chiral Lagrangian of Wilson chiral perturbation theory in the microscopic domain, exact results were obtained for the spectral density of the Hermitian Dirac operator both for the quenched case [4] and the case of dynamical quarks [9]. The spectral density of the nonHermitian Wilson Dirac operator, could only be accessed by means of powerful random matrix techniques. The results for dynamical quarks show that depending on the value of the low-energy constants either an Aoki phase or a first order scenario is possible [10]. Lattice results for the eigenvalue density $[11,12,13]$ have been compared successfully to the exact results $[14,9,15,10$, 16] for the spectral density in the microscopic limit.

Recently, a great deal of attention has been focused on the conformal limit of QCD and QCDlike theories. Both the two-color theory and the any color adjoint theory are relevant for technicolor theories [17]. The advantage of $\mathrm{SU}(2)$ theories is that they require less fermions for achieving conformality than $\mathrm{SU}(3)$ theories. Furthermore, the $\mathrm{SU}(2)$ theory with two adjoint fermions is relevant for minimal walking technicolor theories [18]. Studies of this theory have been performed for unimproved Wilson fermions [19] and for the analysis of the conformal window, it would be useful to have a better understanding of the discretization errors.

As is the case in the continuum theory, also at non-zero lattice spacing, there is a one to one correspondence between patterns of chiral symmetry breaking and the anti-unitary symmetries of the Dirac operator [2]. We can therefore classify QCD in three distinct classes. QCD in the fundamental representation with three or more colors is the case without anti-unitary symmetry. When we have an anti-unitary symmetry, $[T, D]=0$ for the Dirac operator $D$, then there are two different possibilities. Either $T^{2}=1$ or $T^{2}=-1$. In the first case it is always possible to find a gauge field independent basis for which the Dirac operator is real. This is the case for QCD with two colors in the fundamental representation where the chiral symmetry breaking pattern is $\mathrm{SU}\left(2 \mathrm{~N}_{\mathrm{f}}\right) \rightarrow \mathrm{USp}\left(2 \mathrm{~N}_{\mathrm{f}}\right)$. In the second case it is possible to find a gauge field independent basis in which the matrix elements are expressed as self-dual quaternions. This is the situation for QCD in the adjoint representation where the pattern of chiral symmetry breaking is $\mathrm{SU}\left(2 \mathrm{~N}_{\mathrm{f}}\right) \rightarrow \mathrm{SO}\left(2 \mathrm{~N}_{\mathrm{f}}\right)$.

The goal of this paper is to study the effect of a finite lattice spacing on the low lying Dirac eigenvalues and to understand the behavior of the spectral gap of $D_{5}+m \gamma_{5}=\gamma_{5}\left(D_{\mathrm{W}}+m\right)$ at finite quark mass as a function of the lattice spacing. In mean field theory, closure of the spectral gap will serve as an order parameter for the onset of the Aoki phase [4].

This paper is organized as follows. In section 2 we introduce a Wilson Random Matrix Model for SU(2) with fundamental quarks and QCD with adjoint fermions. In particular we consider the $N_{\mathrm{f}}$ flavor partition function as well as the partially quenched partition function. In section 3 , we compare analytical results with Monte Carlo data of the Random Matrix Model. 


\section{Random Matrix Theory}

The random matrix for the Hermitian Wilson Dirac operator proposed in Refs. [4, 20] is given by

$$
D_{5}=\left(\begin{array}{cc}
a A & W \\
W^{\dagger} & a B
\end{array}\right)
$$

where $A$ and $B$ are Hermitian $n \times n$ and $(n+v) \times(n+v)$ matrices, and $W$ is a complex $n \times(n+v)$ matrix. All matrix elements are distributed according to a Gaussian probability distribution. The Random Matrix Theory for $\beta=1$ and $\beta=4$ is obtained by simply choosing real or quaternion matrix elements.

For $a=0$, the Wilson-Dirac operator has $v$ generic zero modes in accordance with the AtiyahSinger index theorem. At finite $a$, one could define the index of the Dirac operator for a fixed gauge field configuration through spectral flow lines or equivalently by [21]

$$
v=\sum_{\lambda_{k}^{W} \in \mathbb{R}} \operatorname{sign}\left(\left\langle k\left|\gamma_{5}\right| k\right\rangle\right),
$$

where the above sum is restricted to the real modes since the eigenfunctions corresponding to complex modes have zero chirality.

The partition function of $D_{5}$ with $N_{\mathrm{f}}$ flavors is given by

$$
Z_{N_{f}}^{\mathrm{RMT}, v}=\int d D_{5} \operatorname{det}^{N_{f}}\left(D_{5}+m \gamma_{5}+z\right) P\left(D_{5}\right),
$$

where $P\left(D_{5}\right)$ is the probability distribution of the matrix elements of $D_{5}$. In the microscopic limit where the combinations $\widehat{m}=2 m n, \widehat{z}=2 z n$ and $\widehat{a}^{2}=a^{2} n / 2$ are kept fixed as $n \rightarrow \infty$, the Random Matrix Theory reduces to the $\varepsilon$-limit of Wilson chiral perturbation theory,

$$
Z_{N_{\mathrm{f}}}^{v}=\int d \mu(U) \operatorname{det}^{\kappa} U \exp \left[\operatorname{tr} \frac{\widehat{m}}{2}\left(U+U^{-1}\right)-\operatorname{tr} \frac{\widehat{z}}{2}\left(U-U^{-1}\right)+\widehat{a}^{2} \operatorname{tr}\left(U^{2}+U^{-2}\right)\right]
$$

with $U \in \mathrm{U}\left(2 N_{f}\right) / \mathrm{Sp}\left(2 N_{f}\right)$ and $\kappa=v / 2$ for $\beta=1$ while $U \in \mathrm{U}\left(2 N_{f}\right) / \mathrm{O}\left(2 N_{f}\right)$ and $\kappa=v$ for $\beta=4$ as is the case for $a=0$ [22].

The full partition function at fixed vacuum angle $\theta$ is given by a sum over the Fourier components $Z_{v}$,

$$
Z(\theta)=\sum_{v=-\infty}^{\infty} e^{i v \theta} Z_{v}
$$

In order to access the spectral properties of the Dirac operator we employ the supersymmetric method of RMT. The generating function for the resolvent of $D_{5}$ is the partially quenched partition function obtained by adding an additional fermionic and bosonic quark to the $N_{f}$ flavor partition function

$$
\mathscr{Z}_{N_{f}+1 \mid 1}^{v}\left(\widehat{m}, \widehat{z}, \widehat{z^{\prime}} ; \widehat{a}\right)=\left\langle\operatorname{det}^{N_{f}}\left(\gamma_{5}\left(D_{\mathrm{W}}+\widehat{m}\right)\right) \frac{\operatorname{det}\left(\gamma_{5}\left(D_{\mathrm{W}}+\widehat{m}\right)+\widehat{z}\right)}{\operatorname{det}\left(\gamma_{5}\left(D_{\mathrm{W}}+\widehat{m}\right)+\widehat{z^{\prime}}\right)}\right\rangle_{v} .
$$

The resolvent of $D_{5}$ is given by

$$
G^{v}(\widehat{z}, \widehat{m} ; \widehat{a})=\lim _{z^{\prime} \rightarrow z} \partial_{z} \mathscr{Z}_{1 \mid 1}^{v}=\left\langle\operatorname{tr} \frac{1}{D_{5}+\widehat{z}}\right\rangle .
$$




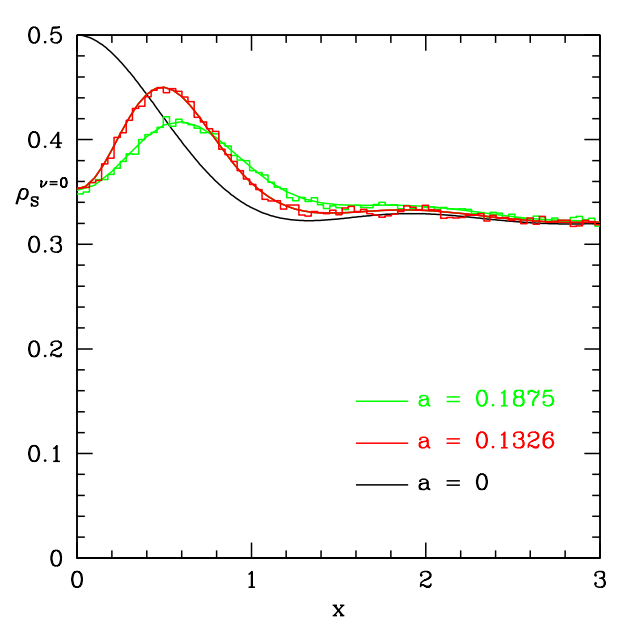

Figure 1: The analytical results (solid curves) compared to the results of the Monte Carlo simulation (histograms).

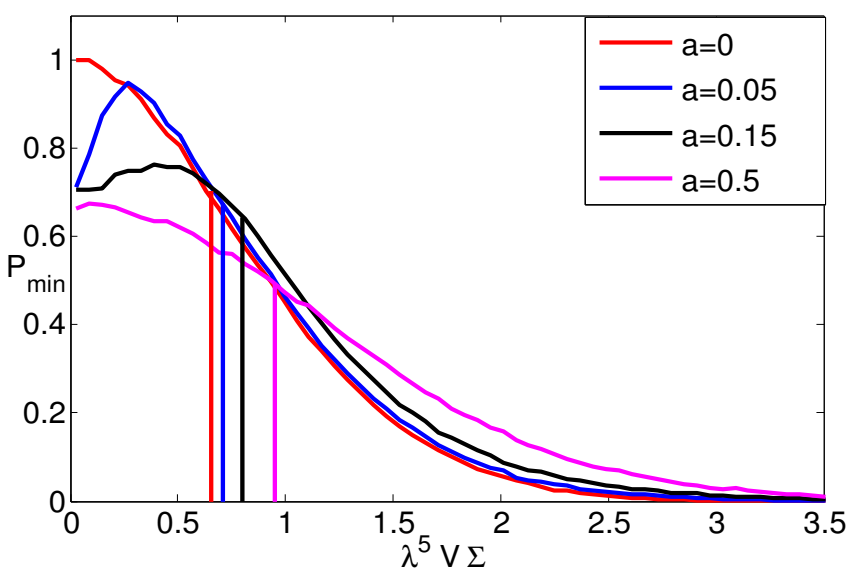

Figure 2: The distribution of the first positive eigenvalue, with $\widehat{m}=0, v=0$. The average position of this eigenvalue (denoted by the vertical bar) shifts away from the origin for increasing $a$.

Its discontinuity across the real axis gives the spectral density

$$
\rho_{5}^{v}\left(\widehat{\lambda}^{5}, \widehat{m} ; \widehat{a}\right)=\frac{1}{\pi} \operatorname{Im}\left[G^{v}\left(\widehat{z}=\widehat{\lambda}^{5}, \widehat{m} ; \widehat{a}\right)\right] .
$$

In the microscopic limit the generating function reduces to a supersymmetric extension of of the partition function (2.4). However the integrals over the non-compact part of $U$ are only convergent for imaginary $a$. To obtain an analytical continuation to real $a$ we have to rotate $U \rightarrow i U$. This results in the partition function

$$
\begin{aligned}
\mathscr{Z}_{N_{\mathrm{f}}+1 \mid 1}^{V} & =\int d \mu(U) \operatorname{Sdet}^{\kappa+N_{\mathrm{f}}} U \exp \left[\frac{i}{2} \operatorname{Str} \widehat{M}\left(U-U^{-1}\right)\right] \\
& \times \exp \left[-\frac{i}{2} \operatorname{Str} \widehat{Z}\left(U+U^{-1}\right)-\widehat{a}^{2} \operatorname{Str}\left(U^{2}+U^{-2}\right)\right] .
\end{aligned}
$$

The integration manifold $U \in \mathrm{U}\left(\mathrm{N}_{\mathrm{f}}+2 \mid 2\right) / \mathrm{UOSp}\left(\mathrm{N}_{\mathrm{f}}+2 \mid 2\right)$ is the same as for $a=0$. The mass matrix is given by $\widehat{M}=\operatorname{diag}(\widehat{m}, \widehat{m}, \widehat{m}, \widehat{m})$ whereas the axial mass is given by $\widehat{Z}=\operatorname{diag}\left(\widehat{z}, \widehat{z}, \widehat{z^{\prime}}, \widehat{z^{\prime}}\right)$. Note that for $z^{\prime} \rightarrow z$ we recover the $N_{\mathrm{f}}$ flavor partition function.

To evaluate this integral we need an explicit parameterization of $U$. Since it involves only four Grassmann variables, it can easily be evaluated by brute force. The results are quite lengthy and will be published elsewhere [23].

\section{Analytical and Numerical results}

To illustrate our analytical results we compare in Fig. 1 the results obtained from (2.9) for $v=0$ and $m=0$ with numerical ones obtained by calculating the eigenvalues of an ensemble of random matrices. Surprisingly, the spectral density at zero decreases by a factor $\sqrt{2}$ for any nonzero value of $a$. The reason for the nonuniformity of the $a \rightarrow 0$ limit is that for $a \neq 0$ the convergence of the integral is achieved through the $U^{2}$-term while for $a=0$ the convergence comes from the 


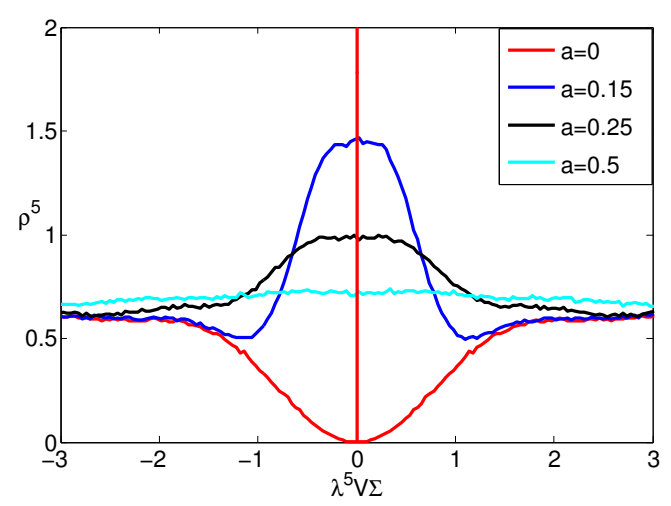

Figure 3: Spectral density $\rho_{5}$ at $v=2$ and $\widehat{m}=$ 0 . Note the presence of zero modes for $a=0$ and the widening of the peak as we increase $a$.

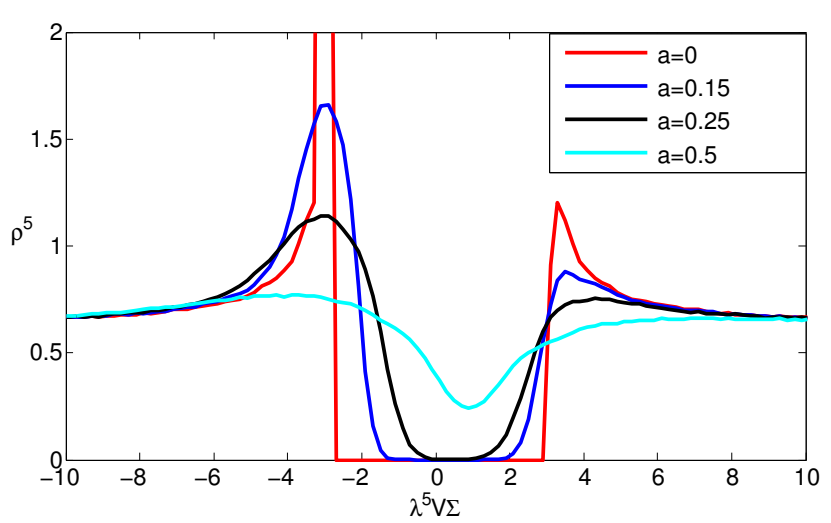

Figure 4: Spectral density $\rho_{5}$ at $v=2$ and $\widehat{m}=3$. For $a=0$ the spectrum has a gap of width $2 m$ which closes for increasing $a$.

$U$-term. The effect of diagonal blocks that comprise the Wilson term in the Random Matrix Model can thus be seen for arbitrarily small values of $a$.

In Fig. 2 we study the distribution of the first positive eigenvalue. Apparently, the diagonal blocks of the Wilson Dirac operator lead to a weak repulsion of the two eigenvalues closest to zero, but for larger values of $a$, there is no repulsion away from zero. The average position of the first eigenvalue increases as is shown by the corresponding vertical bar perpendicular to the real axis.

For $v \neq 0$, the distribution of the zero modes is a Dirac delta function for $a=0$. As we increase the value of $a$ their distribution gets broadened with a width proportional to $a$. This is similar to what happens in QCD with three or more colors in the fundamental representation. As can be seen from Figs. 3 and 4, at about $\widehat{a}=0.5$ the peak due to the would be zero modes has disappeared almost completely.

For $a=0$ the spectrum of $D_{5}$ has a gap $[-m, m]$, but at finite lattice spacing eigenvalues of tail states penetrate the gap [3]. Our results provide an explicit analytical handle on these states and allow us to identify the point where eigenvalues approach the center of the spectral gap and inversion of the Dirac operator becomes very difficult.

For $a=0$, the spectral density of the two-color theory develops a square root type of singularity at the edge of the gap, $\rho_{5}(x) \sim 1 / \sqrt{x^{2}-m^{2}}+v \delta(x-m)$ (see Fig. 4). For $\beta=2$ on the other hand the spectral density approaches a finite limit at $x=m$.

In Figs. 5 and 6 we show scatter plots of the eigenvalues of $D_{\mathrm{W}}$. For $a=0$ the Wilson Dirac operator $D_{\mathrm{W}}$ is anti-Hermitian and the eigenvalues lie on the imaginary axis (see Fig. 5). In contrast to this behavior, $D_{\mathrm{W}}$ is non-Hermitian at finite $a$. Hence, it has a complex spectrum. Because $D_{\mathrm{W}}$ is still $\gamma_{5}$-Hermitian its complex eigenvalues occur in complex conjugate pairs, and at least $|v|$ eigenvalues are real (see Fig. 6). The additional real modes always appear in an even number. In Fig. 6 we show a spectrum of $D_{\mathrm{W}}$ for $v=5$ with seven real modes. 


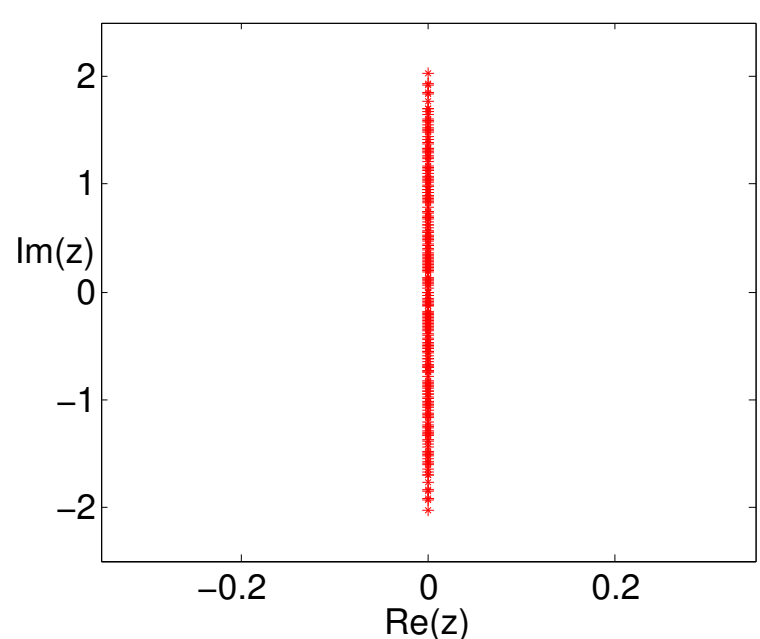

Figure 5: Spectrum of a randomly generated matrix $D_{\mathrm{W}}$ with $v=5$ and $m=0$ at vanishing lattice spacing, i.e. $\widehat{a}=0$.

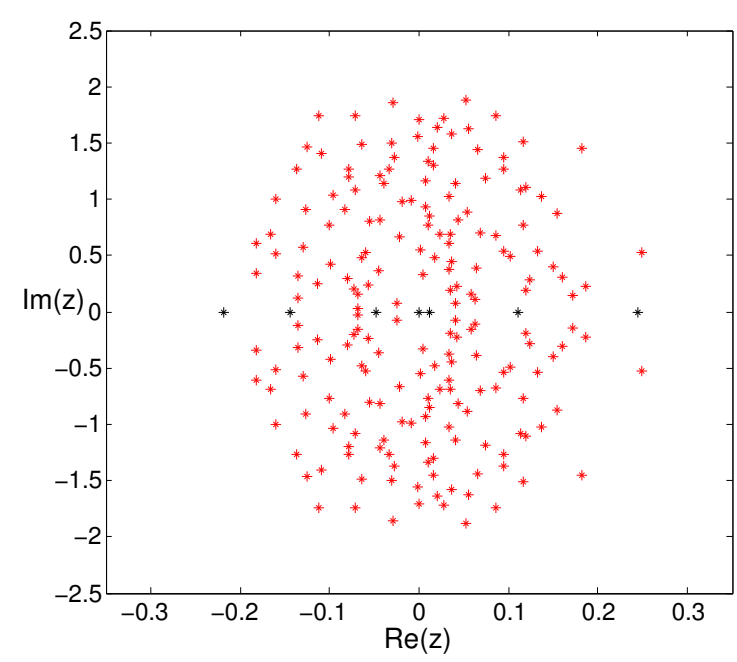

Figure 6: Spectrum of a randomly generated matrix $D_{\mathrm{W}}$ with $v=5$ and $m=0$ for a finite lattice spacing $(\widehat{a}=1)$.

\section{Conclusions}

We have introduced Random Matrix Theories for the Wilson Dirac operator of QCD like theories and have obtained explicit analytical results for the spectral density of two-color QCD. The analytical results for $v=0$ have been compared to Monte Carlo simulations of the proposed random matrix ensemble. Furthermore, numerical results for the spectral density for non-zero quark mass and index $v$ have been presented. We stress that although the increase of computational power has allowed for lattice simulations in the deep chiral regime it is not possible to invert the Wilson Dirac operator when eigenvalues are sufficiently close to zero. Our results identify the parameter domain where such eigenvalues appear and can be potentially useful for identifying the parameter domain of simulations with dynamical quarks. Actually, the probability to obtain small eigenvalues is higher for the two-color theory than for QCD with more colors because of the lack of repulsion from the origin. Our analytical results can be extended to the case of arbitrary $v$ and also to the case of adjoint QCD. These will be presented in forthcoming publications.

Acknowledgments. MK acknowledges financial support by the Alexander-von-Humboldt Foundation. JV and SZ acknowledge support by U.S. DOE Grant No. DE-FG-88ER40388.

\section{References}

[1] E. V. Shuryak and J. Verbaarschot, Random matrix theory and spectral sum rules for the Dirac operator in QCD, Nucl. Phys. A 560 (1993) 306-320, [hep-th/9212088].

[2] J. J. M. Verbaarschot, The Spectrum of the QCD Dirac operator and chiral random matrix theory: The Threefold way, Phys. Rev. Lett. 72 (1994) 2531-2533, [hep-th/ 9401059 ].

[3] J. C. Osborn, D. Toublan, and J. J. M. Verbaarschot, From chiral random matrix theory to chiral perturbation theory, Nucl. Phys. B 540 (1999) 317, [arXiv: hep-th/9806110]. 
[4] P. Damgaard, K. Splittorff, and J. Verbaarschot, Microscopic Spectrum of the Wilson Dirac Operator, Phys. Rev. Lett. 105 (2010) 162002, [arXiv: 1001.2937$].$

[5] J. C. Osborn, Staggered chiral random matrix theory, Phys. Rev. D 83 (2011) 034505, [arXiv:1012.4837].

[6] S. R. Sharpe and R. L. Singleton, Spontaneous flavor and parity breaking with Wilson fermions, Phys. Rev. D 58 (1998) 074501, [hep-lat/9804028].

[7] G. Rupak and N. Shoresh, Chiral perturbation theory for the Wilson lattice action, Phys. Rev. D 66 (2002) 054503, [hep-lat/0201019].

[8] C. Aubin and C. Bernard, Pion and kaon masses in staggered chiral perturbation theory, Phys. Rev. D 68 (2003) 034014, [hep-lat/ 0304014 ].

[9] K. Splittorff and J. J. M. Verbaarschot, The Wilson Dirac Spectrum for QCD with Dynamical Quarks, Phys. Rev. D 84 (2011) 065031, [arXiv:1105.6229].

[10] M. Kieburg, K. Splittorff, and J. J. M. Verbaarschot, The Realization of the Sharpe-Singleton Scenario, Phys. Rev. D 85 (2012) 094011, [arXiv: 1202 . 0620].

[11] P. Damgaard, U. Heller, and K. Splittorff, Finite-Volume Scaling of the Wilson-Dirac Operator Spectrum, Phys. Rev. D 85 (2012) 014505, [arXiv:1110.2851].

[12] A. Deuzeman, U. Wenger, and J. Wuilloud, Spectral properties of the Wilson Dirac operator in the E-regime, JHEP 1112 (2011) 109, [arXiv: 1110.4002 ].

[13] P. Damgaard, U. Heller, and K. Splittorff, New Ways to Determine Low-Energy Constants with Wilson Fermions, [arXiv:1206.4786].

[14] G. Akemann, P. H. Damgaard, K. Splittorff, and J. Verbaarschot, Wilson Fermions, Random Matrix Theory and the Aoki Phase, PoS LATTICE2010 (2010) 092, [arXiv: 1011 . 5118].

[15] M. Kieburg, J. J. M. Verbaarschot, and S. Zafeiropoulos, Eigenvalue Density of the non-Hermitian Wilson Dirac Operator, Phys. Rev. Lett. 108 (2012) 022001, [arXiv:1109.0656].

[16] G. Akemann and T. Nagao, Random Matrix Theory for the Hermitian Wilson Dirac Operator and the chGUE-GUE Transition, JHEP 1110 (2011) 060, [arXiv: 1108 . 3035].

[17] K. Rummukainen, QCD-like technicolor on the lattice, AIP Conf. Proc. 1343 (2011) 51-56, [arXiv:1101.5875].

[18] F. Sannino, Dynamical Stabilization of the Fermi Scale: Phase Diagram of Strongly Coupled Theories for (Minimal) Walking Technicolor and Unparticles, [arXiv:0804.0182].

[19] F. Bursa, L. Del Debbio, L. Keegan, C. Pica, and T. Pickup, Mass anomalous dimension in SU(2) with two adjoint fermions, Phys. Rev. D 81 (2010) 014505, [arXiv: 0910.4535 ].

[20] G. Akemann, P. Damgaard, K. Splittorff, and J. Verbaarschot, Spectrum of the Wilson Dirac Operator at Finite Lattice Spacings, Phys. Rev. D 83 (2011) 085014, [arXiv: 1012 . 0752].

[21] S. Itoh, Y. Iwasaki, and T. Yoshie, The U(1) problem and topological excitations on a lattice, Phys. Rev. D 36 (1987) 527.

[22] A. M. Halasz and J. J. M. Verbaarschot, Universal fluctuations in spectra of the lattice Dirac operator, Phys. Rev. Lett. 74 (1995) 3920, [hep-lat/ 9501025 ].

[23] M. Kieburg, J. J. M. Verbaarschot, and S. Zafeiropoulos, In Preparation (2012).

[24] M. Kieburg, J. J. M. Verbaarschot, and S. Zafeiropoulos, Random Matrix Models for Dirac Operators at finite Lattice Spacing, PoS LATTICE2011 (2011) 312, [arXiv:1110 . 2690]. 